\title{
THE MISSING FLAP: CONSIDERATIONS ABOUT A CASE OF AORTIC INTUSSUSCEPTION
}

Eugenio Neri, MD, Gianni Capannini, MD, Enrico Carone, MD, Enrico Tucci, MD, Francesco Diciolla, MD, and

Carlo Sassi, MD, Siena, Italy

A 51-year-old hypertensive man was admitted with acute thoracic and epigastric pain, which followed a rapidly regressed period of loss of consciousness. The routine diagnostic studies excluded myocardial ischemia or infarction. At the time of hospital admission, neurologic status was normal, with equal carotid and peripheral pulses; a grade 5/6 decrescendo diastolic murmur was heard at the left sternal border. Chest radiograph showed a mediastinal enlargement, and transthoracic two-dimensional echocardiography (Fig 1) revealed the aortic root to be dilated up to $7 \mathrm{~cm}$. No intimal flap could be visualized in the ascending aorta, the aortic valve was massively incompetent, and pericardial effusion was not detectable.

Hemodynamics were stable with no sign of shock. We decided to obtain a contrast computed tomographic (CT) scan to visualize the aorta because we suspected that he had a dissecting aneurysm. The CT scan showed a dilated aortic root with a maximum diameter of $78 \mathrm{~mm}$ (Fig 2). Curvilinear lucencies were present in the supravalvular portion of the aortic root, but such lucencies were absent in the other sections of the ascending aorta. No double lumen was present in the aortic root or the arch. The distal part of the arch was occupied by curvilinear lucencies (Fig 3 ) and the descending aorta was normal. A double lumen was present only in the proximal part of the brachiocephalic artery. Although CT scan findings were unusual for this condition, an aortic type A dissection with intimal intussusception was suspected. We decided to operate on the patient without delay. Meanwhile the neurologic status of the patient deteriorated with the onset of lethargy. A right carotid pulse was still present but reduced in amplitude and bisferiens (reduplicated).

The chest was opened through a median sternotomy and the pericardium was entered. Minimal bloody pericardial effusion was present. The aortic root consisted of only a thin, bluish layer of tissue. On opening of the aorta, the aortic wall, from the level of the sinotubular junction up to the origin of the brachiocephalic trunk, consisted of only the outer layer of media and adventitia. A circumferential tear of the

From the Thoracic and Cardiovascular Department, University Hospital, Siena, Italy.

Received for publication Sept 29, 1998; accepted for publication Nov 17,1998

Address for reprints: Eugenio Neri, MD, Istituto di Chirurgia Toracica e Cardiovascolare Universitá degli Studi de Siena, Policlinico le Scote, Viale M. Bracci, 53100 Siena, Italy.

J Thorac Cardiovasc Surg 1999;117:829-30

Copyright (C) 1999 by Mosby, Inc.

$0022-5223 / 99 \$ 8.00+0 \quad \mathbf{1 2 / 5 4 / 9 5 9 1 6}$

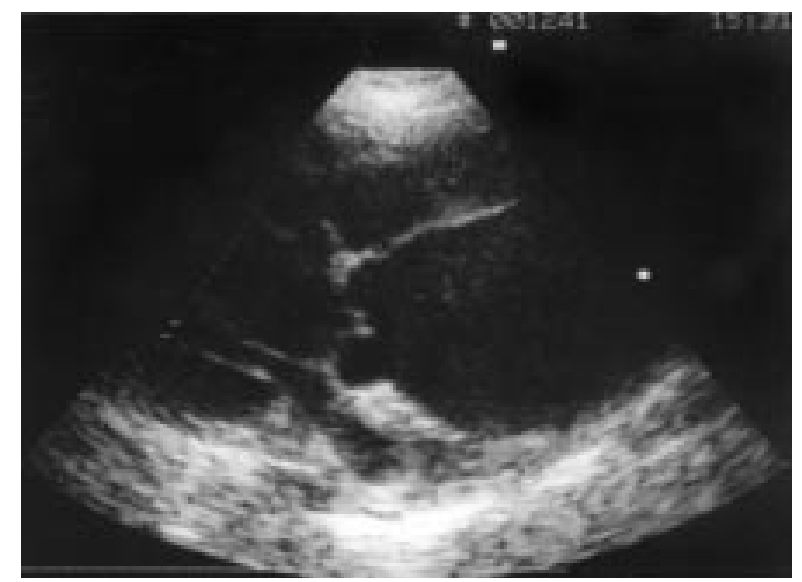

Fig 1. Transthoracic two-dimensional echocardiography revealed a dilated aortic root. No intimal flap could be visualized in the ascending aorta.

intima caused the migration and the intussusception of a tubular flap downstream into the aortic arch and proximal descending aorta. This caused the inability, by CT scan and echocardiography, to detect a double lumen and the typical flap image in the ascending aorta. The flap was in tight contact with the ostia of left carotid and left subclavian arteries, obstructing their flow with a trapdoor mechanism. At the level of the ostium of the brachiocephalic trunk, the tubular flap was partially lacerated, making perfusion of the right carotid and right subclavian arteries possible. Surgical treatment consisted in a Cabrol prosthetic aortic root replacement with a 27-mm composite valve graft (St Jude Medical, Inc, St Paul, Minn). The distal anastomosis was performed with the patient under deep hypothermic circulatory arrest, which allowed the retrieval with the aid of a Foley catheter of the intussuscepted intima from the aortic arch, avoiding any additional laceration. The postoperative course was uneventful and no neurologic deficit was present. The patient recovered well from the operation and was discharged on postoperative day 12. Histologic examination of the aortic wall showed cystic medial necrosis and myxoid degeneration of the aortic valve.

Discussion. Circumferential disruption of the inner aortic wall layers of the ascending aorta, and subsequent distal invagination of the cylindric flap, was first described by Hufnagel and Conrad ${ }^{1}$ in 1962 and named intimo-intimal intussusception. Since then about 20 articles describing cases of intimo-intimal intussusception have been published. ${ }^{2}$ Analysis of the literature reveals that in almost all cases a 


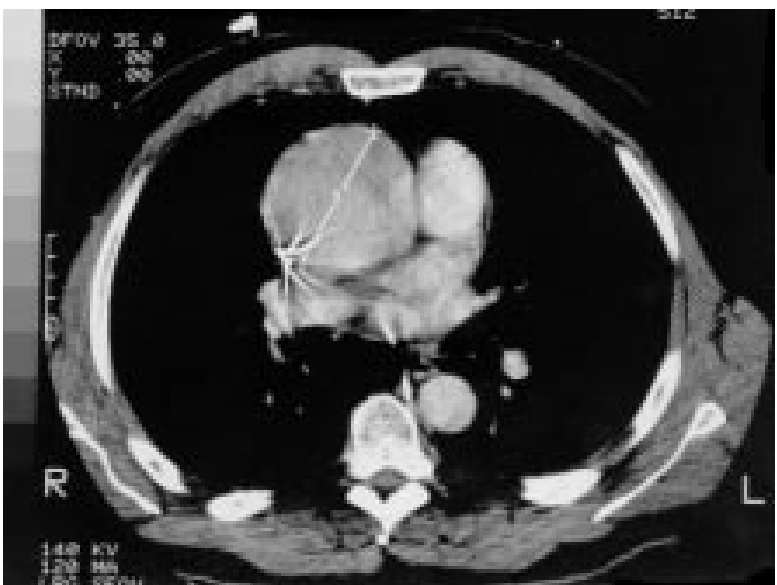

Fig 2. Contrast CT scan showing a dilated aorta without an evident flap. No double lumen is present in the aortic root.

sudden onset of neurologic symptoms has been followed by a short delay of severe chest pain typical of aortic dissection. The neurologic symptoms varied from sudden loss of consciousness to variable degrees of dullness, dizziness, confusion, and restlessness. These symptoms are due to the sudden obstruction of the supra-aortic branches by the intussuscepted intima and are accompanied by lost or reduced carotid pulses. In type A dissection, it can be difficult to differentiate the neurologic symptoms due to the direct dissection of supra-aortic trunks from those caused by direct obstruction by the intussuscepted flap. The sudden onset, the temporal modifications, and the presence of an emerging and deepening of conscience status, accompanied by a variability in carotid pulses, may be regarded as a clue to intimo-intimal intussusception.

CT findings in a patient with intimo-intimal intussusception have been described by Nelsen and coworkers ${ }^{3}$ : enlarged aortic root, mediastinal hematoma, the presence of curvilinear lucencies in the aortic root, the confusing absence of an intimal flap in the mid-ascending aorta, and again the presence of linear lucencies in the aortic arch, are consistent with intussuscepted ascending aortic intima.

Lourié and coworkers ${ }^{4}$ first described an intimo-intimal intussusception detected only by transesophageal echocardiography and considered transesophageal echocardiography to be the gold standard in defining the anatomy of aortic dissection allowing recognition of complex intimal flaps. In a recent article, $\mathrm{we}^{5}$ have reported a case of false positive diagnosis of type A dissection due to the detection by transesophageal echocardiography, in a widened ascending aorta, of the image of an intimal flap. In the same paper we underlined that in emergency settings, such as aortic dissection,

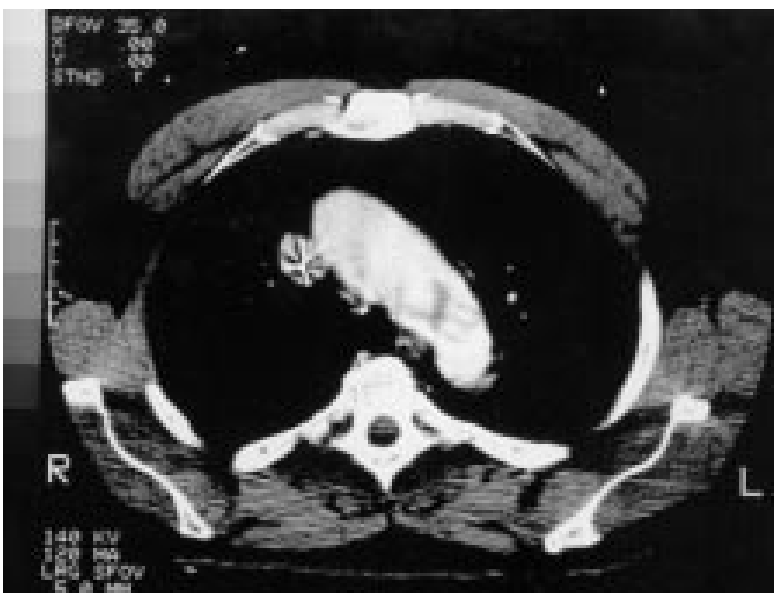

Fig 3. The distal part of the arch is occupied by curvilinear lucencies.

instrumental findings may represent a source of diagnostic pitfalls.

This case of aortic dissection with intimo-intimal intussusception is surprisingly symmetrical, from the diagnostic point of view, and appears as the mirror image of that case: the sharp contrast between the typical clinical presentation of type A dissection and the lack of demonstration, by the common diagnostic tools, of an intimal flap inside a widened ascending aorta may lead to a false negative diagnosis. As evinced from previous reports, the lack of demonstration of an intimal flap in the ascending aorta seems characteristic of this condition and is in marked contrast to the clinical picture of a type A dissection. Thus an undetectable intimal flap is not an absent flap but must be regarded as a "missing" flap.

\section{REFERENCES}

1. Hufnagel CA, Conrad PW. Intimo-intimal intussusception in dissecting aneurysm. Am J Surg 1962;103:723-7.

2. Lijoi A, Scarano F, Canale C, Parodi E, Dottori V, Passerone GC, et al. Circumferential dissection of the ascending aorta with intimal intussusception: case report and review of the literature. Tex Heart Inst J 1994;21:166-9.

3. Nelsen KM, Spizarny DL, Kastan DJ. Intimointimal intussusception in aortic dissection: CT diagnosis. AJR Am J Roentgenol 1994;162:813-4.

4. Lourié JK, Appelbe A, Martin RP. Detection of complex intimal flaps in aortic dissection by transesophageal echocardiography. Am J Cardiol 1992;69:1361-3.

5. Neri E, Maiza D, Bureau G, Lebreton G, Gringore A, Coffin O, et al. False positive diagnosis of ascending aorta dissection by single-plane transesophageal echocardiography. J Thorac Cardiovasc Surg 1996;112:1387-9. 